\title{
Long-term breast cancer studies yield encouraging data for recurrence, survival
}

Susan London reports on 4 pivotal breast cancer studies presented at the 2016 annual meeting of the American Society of Clinical Oncology in Chicago - the MA.17R trial, presented as the plenary talk by Dr Paul Goss, which looked at extending adjuvant aromatase inhibitors to 10 years or beyond in postmenopausal women; two presentations on mutations after progression in metastatic breast cancer, one on first-line Als and the other on prior endocrine therapy (PALOMA-3I); and the Z0011 trial showing that sentinel lymph node dissection without axillary lymph node dissection might show promising 10-year loco-regional control and survival outcomes.

\section{Adjuvant Al therapy for breast cancer: 10 years is superior to 5 years \\ Key clinical point Extending adjuvant Al therapy out to 10 years improves disease-free survival and is generally safe and well tolerated. Major finding Patients who continued taking an $\mathrm{Al}$ out to 10 years had a $34 \%$ lower risk of recurrence or contralateral breast cancer than peers who stopped after 5 years. \\ Data source A randomized placebo-controlled phase 3 trial among 1,918 postmenopausal women who had already completed 5 years of Al therapy (MA. 17R trial). Disclosures Dr Goss disclosed that he had no relevant conflicts of interest. Dr Lemieux disclosed that she had no relevant conflicts of interest. The trial received support from Novartis.}

Extending adjuvant aromatase inhibitor (AI) therapy from 5 years to 10 years further reduces the risk of recurrence and new breast cancer in postmenopausal women treated for early disease, according to findings of the MA. 17R trial. In the phase 3 trial conducted by the Canadian Cancer Trials Group and the North American Breast Cancer Group, 1,918 postmenopausal women who

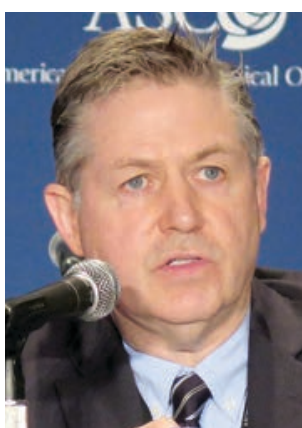

Dr Paul E Goss had completed about 5 years of AI therapy (preceded by tamoxifen in most cases) were randomized to take the AI letrozole or to stop therapy by taking placebo for an additional 5 years. Compared with peers who stopped, women who continued AI therapy for 5 more years had a 34\% lower risk of recurrence or contralateral breast cancer, investigators reported at the meeting. The trade-off was a small increase in the rates of skeletal-related adverse events such as fractures. Quality of life was essentially unaffected.
"MA. 17R is the first study to show the benefit of extending an adjuvant aromatase inhibitor beyond 5 years," commented lead investigator Paul E Goss, MD, director of the Breast Cancer Research Program at the Massachusetts General Hospital and a professor of medicine at Harvard Medical School, both in Boston. "Unlike many anticancer therapies, aromatase inhibitors are readily accessible around the world, and therefore our results will further improve the outcome of many women with breast cancer."

The disease-free survival curves will likely separate further, given a "legacy effect" of endocrine therapy that persists after it ends, he predicted. "Overall survival will eventually become positive in MA.17R. The US Food and Drug Administration has taken the opinion that overall survival follows disease-free survival for endocrine therapies, and I think that's what we see in all the trials."

However, it remains unclear how patients should be managed after 10 years of an AI, according to Dr Goss. "We know from the curve of the natural history of this disease that it chronically relapses, and we continue to see patients 25 years after their primary diagnosis with a recurrence." Some data in mice suggest there may be benefit from continuing the AI until recurrence. "I don't think that would be entered into in clinical practice as a rule, because there is no clinical trial of that," he said. "But this data will now take the aromatase inhibitors out to 10 years."

\section{MA.17R design}

Post-menopausal women were eligible for MA.17R if they had undergone resection of hormone receptor-positive early breast cancer and had already completed about 5 years of therapy with any of the 3 AIs currently on the market. In most cases, they also had previously received tamoxifen. 
Many of the women came from the parent MA.17R trial, which tested an initial 5 years of letrozole against placebo after tamoxifen therapy. Longer-term results of that trial, previously reported (J Clin Oncol. 2012;30:718-21), showed that this duration of letrozole had a significant disease-free survival benefit (hazard ratio, .52 ) and overall survival benefit (HR, .61).

In the MA. 17R trial, the women were randomized to letrozole or a placebo for an additional 5 years, with a primary endpoint of disease-free survival and secondary endpoints including safety and quality of life.

\section{Efficacy and safety}

After a median follow-up of 6.3 years, the 5-year rate of disease-free survival was $95 \%$ with letrozole and $91 \%$ with placebo, according to Dr Goss (simultaneously published, N Eng1 J Med. 2016. June 5 doi: 10.1056/NEJMoa1604700). The difference translated to a more than one-third reduction in the risk of disease recurrence or the occurrence of contralateral breast cancer (HR, .66; $P=.01)$. about threefourths of recurrences were distant.

The groups did not differ significantly with respect to the rate of overall survival, which was $93 \%$ with letrozole and $94 \%$ with placebo. The annual incidence of contralateral breast cancer was sharply lower in the letrozole group, at $0.21 \%$, than in the placebo group, at $0.49 \%$, translating to a more than one-half reduction in this risk of this outcome (HR, .42; $P=.007)$.

No new toxicities or emergent symptoms were noted from extending AI therapy, according to Dr Goss. However, the letrozole group significantly more commonly experienced bone pain ( $18 \%$ vs $14 \%)$, bone fractures (14\% vs $9 \%)$, and new-onset osteoporosis (11\% vs 6\%). Therefore, "bone health remains important for risk-benefit consideration," he said.

\section{Patient-reported outcomes}

In a separate session and the press briefing, Dr Julie Lemieux, a researcher at the Centre Hospitalier affilié Universitaire de Québec, reported the trial's patientreported outcomes, ascertained from questionnaires completed at baseline and annually out to 5 years.

In general, both the letrozole and placebo groups had small deteriorations over time in global quality of life as assessed from summary scores on the mental and physical scales of the 36-item Short Form Health Survey (SF-36). But both also had small improvements over time in scores on the Menopause-Specific Quality of Life scale.

When compared, the 2 groups were statistically indistinguishable on most of these measures. The only significant difference was greater worsening in the letrozole group on the role function-physical subscale of the SF-36, which pertains to difficulty performing work or physical activity due to physical health. However, the difference averaged just 3.2 points, which fell short of the 5 points that the investigators considered clinically important.

"The limitation of this analysis was that it was a highly selected population. All of these women had already tolerated 5 years of an aromatase inhibitor, and about $70 \%$ had received 5 years of tamoxifen before," Dr Lemieux commented. "Also, they were clinical trial participants."

Nonetheless, these findings "are very reassuring for those women who want a longer duration of adjuvant endocrine therapy that they can expect a preserved quality of life," she concluded.

\section{ESR 1 mutations found prognostic but not predictive in metastatic breast cancer Key clinical point An ESR 1 mutation in circulating DNA} after progression on endocrine therapy was a marker for poor prognosis but did not predict benefit from subsequent therapy.

Major finding Women with ESRI mutations had poorer progression-free and overall survival (hazard ratios, 1.7 and 1.9). Adding palbociclib to fulvestrant halved the risk of progression-free survival events, regardless of the presence of an ESR 1 mutation. Data source A retrospective cohort study of 144 women with metastatic breast cancer who had experienced progression on a first-line aromatase inhibitor, and an analysis of 395 women with advanced breast cancer from a randomized trial testing addition of palbociclib to fulvestrant after progression on prior endocrine therapy (PALOMA-3 trial).

Disclosures Dr Clatot disclosed that he receives research funding from Novartis. Dr Turner disclosed that he receives honoraria from and has a consulting or advisory role with AstraZeneca, Pfizer, and Roche Pharma; Pfizer sponsored PALOMA-3, and AstraZeneca provided the fulvestrant.

Mutation of the estrogen receptor 1 (ESR1) gene, one of the mechanisms whereby tumors become resistant to endocrine therapy, may be prognostic but not predictive in women with hormone receptor-positive advanced breast cancer that has progressed on this therapy, two studies showed.

In a cohort of women who had experienced progression on a first-line aromatase inhibitor, those with ESR1 mutations detected in circulating cell-free DNA at the time of progression had a $70 \%$ higher risk of progression-free survival events and a $90 \%$ higher risk of death thereafter. But the presence of this mutation did not predict benefit from subsequent therapy.

Similarly, in an analysis of women who had experienced progression on prior endocrine therapy and were treated on the randomized PALOMA-3 trial with fulvestrant plus either palbociclib (a cyclin-dependent kinase inhibitor) or placebo, adding the drug reduced the risk of progressionfree survival events similarly, by about $50 \%$, regardless of the presence of ESR1 mutations before starting therapy.

\section{Findings in context}

These new findings can help guide decisions about which 
patients should be tested for ESR1 mutations, according to invited discussant Sarat Chandarlapaty, $\mathrm{MD}, \mathrm{PhD}$, a medical oncologist at the Memorial Sloan Kettering Cancer Center in New York said at the meeting. "Putting it all together, we see ESR1 mutations arise in the metastatic setting subclonally with prolonged exposure to low-estrogen environments," he said. "If we are going to do testing, it makes sense to do it in the setting in which there is prior exposure to an aromatase inhibitor in metastatic ER-positive breast cancer."

The studies' results also help clarify what the finding of an ESR1 mutation means for patient prognosis and choice of next therapy, Dr Chandarlapaty said. "It's clear from two large studies that ESR1 mutation prognosticates poorer and shorter survival, so just the finding alone may aid as sort of a clinical risk assessment for physicians. For the question of prediction, I would say the weight of evidence - even though the clinical studies are small -all the way from biology to clinic is that ESR1-mutant patients are unlikely to benefit from a second-line aromatase inhibitor."

However, "the question of whether testing should be made available in practice on the basis of this particular clinical decision is more complicated," Dr Chandarlapaty said. "For one, is second-line aromatase inhibitor alone a widely used option? Second, does the adoption now of palbociclib in the first-line setting change the biology and the nature of resistance at this later line - in other words, are we going to see patients going on to a second-line aromatase inhibitor after they've had a prior aromatase inhibitor plus palbociclib?"

\section{Mutations after progression on first- line aromatase inhibitors}

In the first study, Florian Clatot, $\mathrm{MD}, \mathrm{PhD}$, a medical oncologist at the Centre Henri Becquerel, University of Normandy, Rouen, France, and colleagues retrospectively studied 144 women who had experienced progression on a first-line aromatase inhibitor.

The investigators used digital droplet polymerase chain reaction (PCR) testing to screen for four ESR-1 mutations in circulating cell-free DNA collected before, at the time of, and after the progression.

Overall, $30.6 \%$ of women were found to have at least one of these mutations at the time of progression, Dr Clatot reported. The prevalence was higher the longer women had been on the aromatase inhibitor.

After progression, most women went on to receive chemotherapy or alternative endocrine therapy with either the selective estrogen receptor modulator tamoxifen or the estrogen receptor antagonist fulvestrant. With a median follow-up of 40 months, multivariate analyses showed that the group with ESR1 mutations at progression had higher risks of subsequent progression-free survival events (hazard ratio, $1.7 ; P=.008$ ) and death (hazard ratio, 1.9; $P=.002$ ).
However, the mutations were not predictive: Women having one fared more poorly, whether given chemotherapy or given tamoxifen or fulvestrant.

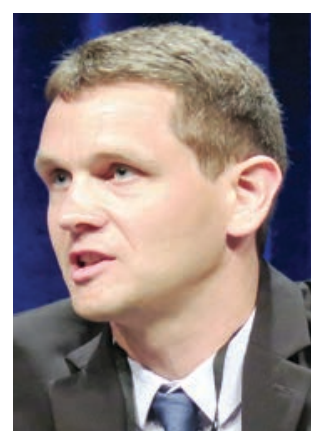

Dr Florian Clatot
Kinetic analyses showed that $75 \%$ of the ESR1 mutations seen at progression were already detectable in the 3 and even 6 months before that event. "Most of the mutations detected before progression increased while aromatase inhibitor therapy was ongoing," Dr Clatot commented. "These results suggest that the preclinical detection of ESR1 circulating mutation may [be of] clinical interest."

Most women who had mutations at progression saw a decrease in the amount detectable over the subsequent 3 months with therapy, including to the point of not being detectable in about half of cases with a reduction. All of those having an increase in mutational burden had progression on their next therapy, compared with only about $40 \%$ of those having a decrease in burden.

"Taken together, these results suggest that the selection pressure provided by aromatase inhibitor exposure is one of the main mechanisms of ESR1 mutation increase," concluded Dr Clatot. "ESR1 mutations are a strong and independent marker of poor prognosis but do not have any predictive value with the treatments used in our cohort."

Mutations after progression on prior endocrine therapy In the second study, Nicholas C Turner, $\mathrm{MD}, \mathrm{PhD}$, a medical oncologist at the Royal Marsden Hospital and Institute of Cancer Research, London, and colleagues analyzed data from a subset of 395 women from PALOMA-3.

The randomized phase 3 trial tested fulvestrant combined with palbociclib, an oral inhibitor of cyclin-dependent kinases 4 and 6 , or placebo. All of the women enrolled had experienced progression on prior endocrine therapy in the adjuvant, advanced, or metastatic setting.

The investigators looked for

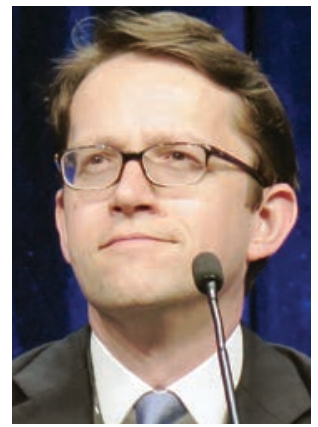

Dr Nicholas C. Turner 12 ESR1 mutations in circulating tumor DNA from baseline plasma samples using the BEAMing (beads, emulsion, amplification, magnetics) digital PCR technique and droplet digital PCR screening.

Overall, $27 \%$ of the women had ESR1 mutations before starting therapy on the trial, Dr Turner reported. Mutations were seen in those who had received a prior aromatase inhibitor, but not in those who had received only 
prior tamoxifen. "So it's quite clear these mutations are not a mechanism of resistance to tamoxifen, suggesting that tamoxifen must have at least some activity against these mutations," he commented.

In addition, ESR1 mutations were more common in patients who had been sensitive versus not to prior endocrine therapy of any type (30.3\% vs $12.8 \%)$ and in patients who had been sensitive versus not specifically to prior aromatase inhibitor therapy (34.6\% vs $11.1 \%$ ).

Stratified analyses showed that palbociclib was similarly superior to placebo in terms of progression-free survival whether patients were positive for an ESR1 mutation (9.4 vs 4.1 months; hazard ratio, $0.52 ; P=.0052$ ) or negative (9.5 vs 3.8 months; hazard ratio, 0.44 ; $P$ less than .0001).

"Detection of estrogen receptor mutations was strongly associated with acquired resistance to prior aromatase inhibitors," Dr Turner said. "Palbociclib offered high efficacy regardless of the estrogen receptor mutation status. And because in this patient population estrogen receptor mutations are detected frequently, the combination of palbociclib and fulvestrant presents an attractive treatment option for patients who have been previously treated with and progressed on aromatase inhibitors."

\section{Safety of sentinel node dissection alone holds up a decade out \\ Key clinical point In women with clinical early-stage breast} cancer who have a positive sentinel node and undergo breast-conserving therapy, skipping ALND does not compromise outcomes. Major finding Women treated with and without ALND were statistically indistinguishable with respect to 10 -year rates of locoregional recurrence $(6.2 \%$ and $5.3 \%)$, disease-free survival $(78.2 \%$ and $80.2 \%)$, and overall survival (83.6\% and $86.3 \%$ ). Data source A randomized phase 3 trial among 891 women with clinical T1-2,N0,MO breast cancer and positive sentinel nodes treated with breast-conserving therapy and usually adjuvant systemic therapy (ACOSOG ZOO 1 1). Disclosures Dr Giuliano disclosed that he had no relevant conflicts of interest.

Women with clinical early-stage breast cancer and a positive sentinel lymph node who receive breast-conserving therapy can safely skip an axillary lymph node dissection (ALND), and therefore avoid its associated morbidity, confirms long-term follow-up of the Z0011 trial conducted by the American College of Surgeons Oncology Group and the Alliance for Clinical Trials in Oncology.

The phase 3 trial enrolled 891 women with clinical T1-2,N0,M0 disease who underwent lumpectomy and were found to have sentinel node involvement. They were randomized to ALND or no further surgery, followed by whole-breast radiation therapy and, in most cases, systemic adjuvant therapy.

The trial was closed early because of low rates of accrual and events. Results at 6.3 years of follow-up showed that compared with peers who had an ALND, the women who skipped this surgery did not have inferior 5-year rates of locoregional recurrence or overall survival (JAMA. 2011;305:569-75).

"The study, however, like most breast cancer studies, con-

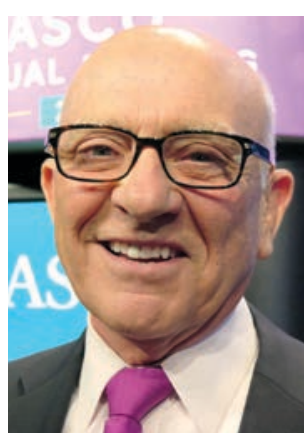

Dr Armando Giuliano tained mostly postmenopausal women with hormone receptorpositive tumors who are known to have late recurrences, and it was criticized for short followup," said first author Armando E Giuliano, MD, of the surgical oncology in the department of surgery, and associate director of surgical oncology in the Samuel Oschin Comprehensive Cancer Institute, Los Angeles. In an update of the findings, now with a median follow-up of 9.3 years, the groups were statistically indistinguishable with respect to 10-year rates of the same outcomes, he reported at the meeting.

"This study ... shows that sentinel node biopsy alone provides excellent 10 -year locoregional control and survival comparable to completion axillary lymph node dissection for these patients, even with long-term follow-up," he maintained. "Routine use of axillary lymph node dissection should be abandoned."

"This was designed as a noninferiority trial, and I would suggest that based on the data we have seen, even if they had hit their target accrual, the outcomes would not be different," said invited discussant Elizabeth A Mittendorf, $\mathrm{MD}, \mathrm{PhD}$, of the University of Texas MD Anderson Cancer Center, Houston. "Clearly, even before today's presentation, Z0011 has been identified as a practice-changing trial, as evidenced by the NCCN guidelines."

In fact, a study last year showed

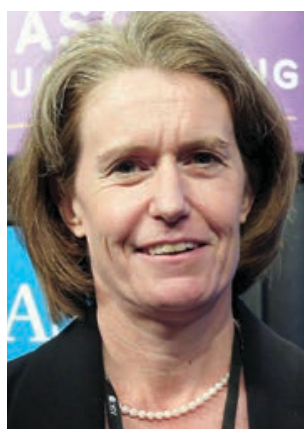

Dr Elizabeth A. Mittendorf that among patients in the general US population meeting the trial's enrollment criteria, the use of sentinel lymph node dissection alone has increased significantly since the Z0011 results were first reported (J Am Coll Surg. 2015;221:71-81).

"However, I would highlight that we have also seen an increase in omission of axillary lymph node dissection for patients who do not meet the Z0011 criteria to include those not planned for radiotherapy, those receiving APBI [accelerated partial breast irradiation], and those undergoing mastectomy," she added. "I highlight these examples specifically because it's been suggested that one of the reasons the patients on the trial have outstanding regional 
control is because of the radiation administered as part of their breast-conserving therapy."

"We will obtain additional data on the locoregional management of these early-stage patients with clinically nodenegative breast cancer," Dr Mittendorf predicted, pointing to the similar POSNOC trial (which is comparing systemic therapy with versus without axillary treatment) and SOUND trial (which is comparing sentinel node dissection versus no axillary surgery).

In Z0011, all women had tumor in sentinel nodes detected with hematoxylin and eosin staining. Those with sentinel node tumor detected only by immunohistochemistry were excluded, as were those who had matted nodes, three or more involved sentinel nodes, or planned thirdfield (nodal) irradiation.

Overall, 27.4\% of the patients in the ALND group had additional positive nodes removed beyond their sentinel nodes. "There is no reason to suspect that women with sentinel node biopsy [only] had fewer involved nodes than the women treated with axillary lymph node dissection," Dr Giuliano commented; thus, a similar share of the former group likely had residual axillary disease that went unresected.

The updated findings showed that the women received ALND and the women who did not were statistically indistinguishable with respect to the 10 -year rate of locoregional recurrence (6.2\% and 5.3\%). Of note, only a single regional recurrence was seen after the initial 5 years of follow-up, and it occurred in the group that did not have ALND.

The groups treated with and without ALND were also statistically indistinguishable with respect to 10 -year rates of disease-free survival ( $78.2 \%$ and $80.2 \%)$, locoregional recurrence-free survival $(81.2 \%$ and $83.0 \%)$, and overall survival ( $83.6 \%$ and $86.3 \%)$.

In multivariate analysis, omission of ALND did not significantly predict locoregional recurrence or overall survival, reported Dr Giuliano. In addition, stratified analysis showed that the lack of difference in overall survival between study groups was the same whether tumors had hormone receptors or not.

In a related analysis of radiation protocol deviations in a subset of women from the trial, $11 \%$ did not receive any radiation therapy, while $18.9 \%$ received third-field radiation, with equal distribution of the latter between study groups (J Clin Oncol. 2014;32:3600-3606). Omission of radiation was associated with an increased risk of local recurrence and death, but it did not affect nodal recurrences. Receipt of third-field radiation did not influence survival. 